\title{
Review of Neutron Diagnostics Based on Fission Reactions Induced by Fusion Neutrons
}

\author{
Urszula Woźnicka ${ }^{1}$ (D) \\ Published online: 14 August 2018 \\ (C) The Author(s) 2018
}

\begin{abstract}
Neutrons are an exceptional diagnostic signature in magnetic fusion experiments with high temperature plasma. A part of neutron detection methods relies on detecting nuclear reactions products, initiated by neutrons. Among different possibilities, the neutron-induced fission reactions can be used. These methods use the specific properties of the radioactive decay phenomena induced by neutrons of heavy elements like uranium or thorium. The neutron-induced fission reactions produce energetic fission fragments that can be detected in a special type of ionization chambers that has one of electrode covered with fissionable material. A fission reaction may be also adapted to neutron detection by activation method. A dedicated target-sample made from fissionable isotope (like ${ }^{235} \mathrm{U},{ }^{238} \mathrm{U},{ }^{232} \mathrm{Th}$ ), irradiated by neutrons, is the source of prompt and delayed neutrons. The decay curve of delayed neutrons can be registered almost immediately after irradiation by fusion neutrons, with a delay resulting exclusively from the time of transport of the samples to the measuring set-up. The yield of a fusion neutron source can be calculated from that measurement. Fission phenomena and fissionable materials are applicable in plasma neutron diagnostics. The lecture gives the introduction to the nuclear fission process and the particular attention is done to the phenomenon and physics of delayed neutrons which are generated during the fission reaction. Examples of delayed neutrons activation set-up and fission chambers dedicated for tokamaks are presented.
\end{abstract}

Keywords Thermonuclear fusion diagnostics - Fission reactions - Delayed neutrons - Neutron activation method . Fission chambers

\section{Introduction}

Neutrons resulting from the thermonuclear fusion of hydrogen isotopes (deuterium and/or tritium, DD, DT) have properties characteristic for a given nuclear (fusion) reaction. For this reason, neutron measurements are a valuable measure of the course of nuclear reactions in plasma. The principal objective of neutron diagnostics is to characterize the fusion plasma under study as a neutron source. In deuterium plasma or deuterium-tritium mixture plasma mainly 2.5 and $14 \mathrm{MeV}$ neutrons are generated from the nuclear reactions ${ }^{2} \mathrm{H}\left({ }^{2} \mathrm{H}, \mathrm{n}\right){ }^{3} \mathrm{He}$ and ${ }^{3} \mathrm{H}\left({ }^{2} \mathrm{H}, \mathrm{n}\right){ }^{4} \mathrm{He}$. Some neutrons can be generated from nuclear reactions between light ions $\left(\mathrm{p},{ }^{2} \mathrm{H},{ }^{3} \mathrm{He}\right)$ and plasma impurities: light

Urszula Woźnicka

Urszula.Woznicka@ifj.edu.pl

1 Institute of Nuclear Physics, Polish Academy of Sciences, Radzikowskiego 152, Kraków 31-342, Poland plasma ions accelerated to high energies $(>1 \mathrm{MeV})$ produce neutrons over a broad energy range. Neutrons can be generated also from photonuclear reactions, as an effect of an acceleration of plasma electrons at high energies $(>10 \mathrm{MeV})$ which generate further $(\gamma, \mathrm{n})$ reactions.

Neutron measurement methods that are possible in fusion reactors experiments (tokamaks, etc.) are restricted by the difficult access to the plasma vessel and a harsh environment caused by strong mixed radiation fields, strong magnetic fields and currents, powerful high frequency wave generators and power supply, heat loads, etc.

Diagnostics of fusion neutrons provide an indicator of plasma properties [1], like fusion power, ion temperature profile, deuterium and tritium energy spectra, incidence of fast particles, neutron source profile and fluence, fuel ion ratio (tritium-to-deuterium) in plasma core, etc. In particular, neutron data will be instrumental for the real-time control of fusion power in future reactors. Detection systems are built on the basis of neutron cameras, monitors, 
spectrometers as well as fission chambers and neutron activation systems.

Since the neutrons are electrically neutral particles they have usually to be converted into charged ones to be detected. Generally, a neutron detector must be equipped with a converter (to convert neutron to detectable radiation) and with a conventional radiation detector (e.g. gaseous, semiconductor, scintillation). Two basic types of neutron interaction with matter are suitable for this conversion: neutron scattering and absorption. Neutron absorption reaction is a common method of neutron detection. This mainly bases on radiation capture and nuclear fission. The neutron is absorbed by target material (converter) emitting secondary particles such as protons, alpha or beta particles, photons or fission fragments. Some reactions are threshold reactions requiring a minimum energy of neutrons. The most common isotopes for the neutron converting material are presented in Table 1 with the corresponding crosssections and abundances in the natural elements [2].

Although fissile isotopes have significantly lower fission cross-sections compared to the absorption cross-sections of other isotopes used as neutron converters (Table 1), they have other unique properties that can be used to detect neutrons. The fission fragments are heavy charged particles. That fission fragments create a high output signal, because they deposit a large amount of energy in a detector sensitive volume. This allows an easy discrimination of the background radiation, e.g. gamma ray.

- Uranium-lined ion chambers (fission chambers) are common neutron sensors employed to monitor intense sources of neutrons e.g. in fusion devices.

The method bases on registering specific products of fission reactions-delayed neutrons. The rate of decay of delayed neutrons can be registered by conventional neutron counters and next recalculated to the primary neutron flux which reached the fission sample. The method has application for neutron sources of very high fluxes.
- Samples of selected uranium isotopes can be used in activation methods to determine a neutron fluence or an energy range of the neutron flux, using selected threshold reactions.

\section{Nuclear Fission Reaction and Fission Chamber for Neutrons Detection}

In nuclear physics and nuclear chemistry, nuclear fission refers to either a nuclear reaction or a radioactive decay process in which the nucleus of an atom splits into smaller parts (lighter nuclei), often producing free neutrons and photons. Fission reaction is mainly induced by neutrons but also spontaneous radioactive decay (not requiring a neutron) is also referred to as fission, and occurs especially in very high mass number isotopes (See, e.g. [3-5]).

Some nuclides are capable of undergoing fission after capturing a neutron and the fission cross section on that reaction depends on the incident neutron energy (Table 1 and Fig. 1). Nuclides like ${ }^{235} \mathrm{U},{ }^{233} \mathrm{U}$, and ${ }^{239} \mathrm{Pu}$ are fissioned with thermal neutrons, e.g. the fission cross section of ${ }^{235} \mathrm{U}$ is $575 \mathrm{~b}$ for incident neutron energy $0.025 \mathrm{eV}$ and strongly goes down with neutron energy because the binding energy resulting from the absorption of a neutron is greater than the critical energy required for fission. Among them only ${ }^{235} \mathrm{U}$ is present in natural uranium $(0.71 \%)$. By a contrast, the binding energy released by ${ }^{238} \mathrm{U}$ (with $99 \%$ of abundance) which absorbed a thermal neutron is less than the critical energy, so the neutron must be faster and having higher energy to realize a fission reaction. For ${ }^{238} U$ the characteristic threshold of incident neutron energy is about $1 \mathrm{MeV}$ and achieves value about $1 \mathrm{~b}$ for neutron energy (2.45-14) MeV. Figure 1 presents the fission cross sections in the function of neutron incident energy for ${ }^{238} \mathrm{U},{ }^{235} \mathrm{U}$ and for ${ }^{232} \mathrm{Th}$. That last isotope characterizes by the sharp energy threshold on $500 \mathrm{keV}$ and fission cross section in the neutron energy range (2.45-14) $\mathrm{MeV}$ is about $0.5 \mathrm{~b}$. The presence of the energy threshold is a valuable feature

Table 1 Most common isotopes use as neutron absorbers applied in neutron detectors

\begin{tabular}{lll}
\hline Neutron induced reaction & Capture/fission cross section $\sigma(\mathrm{b})$ & Abundance of the isotope in the natural element $(\%)$ \\
\hline${ }_{5}^{10} \mathrm{~B}(\mathrm{n}, \alpha){ }_{3}^{7} \mathrm{Li}$ & 3837 (thermal neutrons) & 19.9 \\
${ }_{2}^{3} \mathrm{He}(\mathrm{n}, \mathrm{p}){ }_{1}^{3} \mathrm{H}$ & 5333 (thermal neutrons) & 0.0137 \\
${ }_{3}^{6} \mathrm{Li}(\mathrm{n}, \alpha){ }_{1}^{3} \mathrm{H}$ & 940 (thermal neutrons) & 7.5 \\
${ }_{48}^{113} \mathrm{Cd}(\mathrm{n}, \gamma){ }_{48}^{114} \mathrm{Cd}$ & 20,615 (thermal neutrons) & 12.22 \\
${ }_{92}^{235} \mathrm{U}(\mathrm{n}$, fission $)$ & $\sim 582$ (thermal neutrons) & 0.72 \\
${ }_{92}^{238} \mathrm{U}(\mathrm{n}$, fission $)$ & $\sim 1(2.45-14) \mathrm{MeV}$ & 99.27 \\
${ }_{90}^{232} \mathrm{Th}(\mathrm{n}$, fission $)$ & $\sim 0.5(2.45-14) \mathrm{MeV}$ & 100 \\
\hline
\end{tabular}




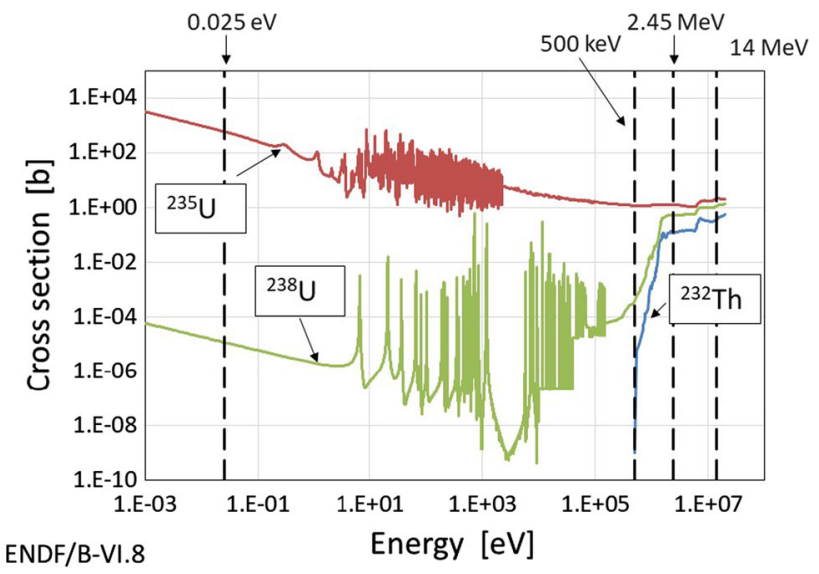

Fig. 1 Fission cross-sections for ${ }^{235} \mathrm{U},{ }^{238} \mathrm{U}$ and ${ }^{232} \mathrm{Th}$ from ENDF/BVI.08 library at $300 \mathrm{~K}$ [6]. Dashed lines indicate characteristic energy levels of neutrons: thermal neutrons $(0.025 \mathrm{eV})$, energy threshold for

${ }^{232} \mathrm{Th}(500 \mathrm{keV})$, neutron energy for DD reaction $(2.45 \mathrm{MeV})$, and neutron energy for DT reaction $(14 \mathrm{MeV})$

for the detection of fast neutrons. Both ${ }^{238} \mathrm{U}$ and ${ }^{235} \mathrm{U}$ isotopes are characterized by the presence of multiple resonant lines in the area below $500 \mathrm{keV}$. This should be taken into account when designing systems for fast neutron measurements with these isotopes and provide adequate shields against neutrons from the resonant region.

Any combination of lighter nuclei can be produced during neutron induced fission reaction, but the most common event is a slightly unequal fission in which one daughter nucleus has a mass of about 90 to $100 \mathrm{u}$ (uatomic mass unit) and the other the remaining 130-140 u [4]. Unequal fissions are energetically more favorable because it allows one product to be closer to the energetic minimum near mass $60 \mathrm{u}$ (only a quarter of the average fissionable mass), while the other nucleus with mass $135 \mathrm{u}$ is still not far out of the range of the most tightly bound nuclei. Distribution of the mass of products depends on the energy of incident neutrons and on the kind of the parent nucleus. The much known two-hump curves of mass distributions of fission fragments after exposure of ${ }^{233} \mathrm{U}{ }^{235} \mathrm{U}$ and ${ }^{139} \mathrm{Pu}$ with thermal neutrons are presented in Fig. 2. It is noteworthy that the mass distributions differ slightly for different isotopes. These differences can be important in the design of fission detectors in which the signal is formed by ionized fission products.

The neutron induced fission reactions can be directly used for a neutron detection via fission products. They carry high kinetic energy, about $160 \mathrm{MeV}$. Thank to this, the fission detector is characterized by a very strong signal originating from fission products and weak disturbances from any competitive reactions or other events generating the measurement background.

The most common form of fission detector [7] is a cylindrical ionization chamber which consists of a pair of

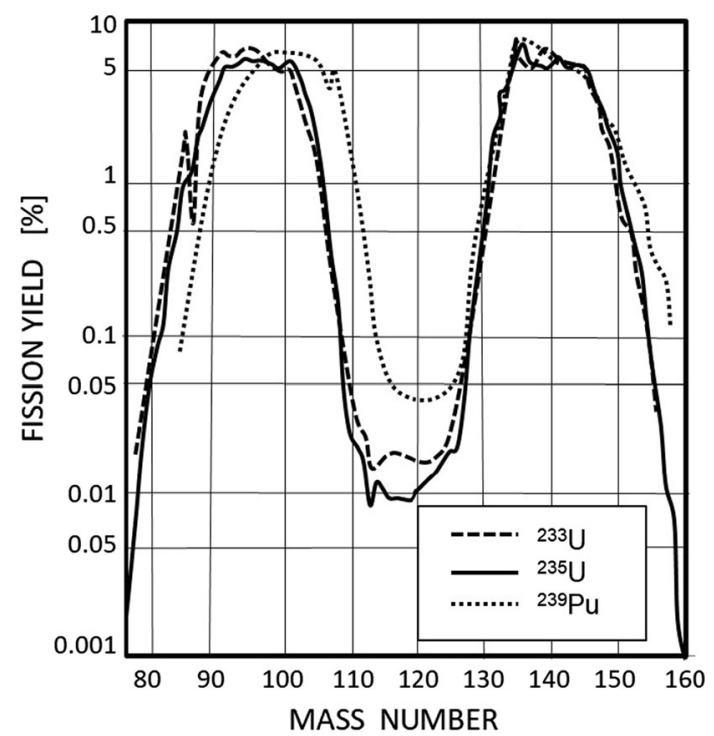

Fig. 2 Characteristic course of the two-hump distribution curve of fission fragments (after exposure with thermal neutrons) with a visible shift for ${ }^{239} \mathrm{Pu}$ in relation to ${ }^{233} \mathrm{U}$ and ${ }^{235} \mathrm{U}$

electrodes, one of this is coated with the fissile deposit, e.g. ${ }^{235} \mathrm{U},{ }^{238} \mathrm{U}$ or ${ }^{242} \mathrm{Pu}$. The detector is filled with the inert gas, mainly with argon and small other additions. Fission chambers can operate in pulse and/or current mode depending on the dynamic range of measurement. The signal of a fission chamber depends primarily on the fissile deposit thickness and the geometric conditions under which the fission fragments are collected. In the case of deposits that are very thin compared with fragments range, a double-humped fission fragments energy spectrum is observed as the detector response with maxima at approx. 70 and $100 \mathrm{MeV}$, with a shape analogous to that shown in Fig. 2. If the deposit is made thicker to enhance detection efficiency, the energy loss of fragments within the deposit will reduce the average fragment energy and distort the shape of measured distribution.

Fission chambers are robust neutron detectors (can operate under high temperatures and under electromagnetic loads). Normally highly enriched ${ }^{235} \mathrm{U}$ is used for the coating of fission chamber electrodes, which makes fission chamber sensitive mainly to thermal neutrons. Another coatings of threshold fissionable isotopes, e.g. ${ }^{238} \mathrm{U}$ can be used to detect fast neutrons. It is expected that large devices dedicated to thermonuclear fusion research, such as ITER [1, 8] will be equipped with fission detectors to measure of neutron yield of DD od DT fusion plasma. Knowledge of neutron source strength is essential for measurement of a total fusion power generated by tokamak. Fission detectors will be the important diagnostic for ITER operation and the neutron emission rate measured by fission chambers will be used as a feedback parameter for fusion output control in ITER. 
For example, a set of fission chambers were used for neutron flux measurements in TFTR [9] tokamak fusion plasma experiments. Both types of fissionable materials threshold $\left({ }^{238} \mathrm{U},{ }^{232} \mathrm{Th}\right)$ and without threshold $\left({ }^{235} \mathrm{U}\right)$ were used in the detectors for neutron flux measurement. ${ }^{235} \mathrm{U}$ fission chambers were covered by neutron moderators to improve sensitivity and detector response function. The different mass of fissionable material was used to cover the measurement possibilities for a wide range of neutron yield (from $10^{10}$ to $>10^{19} \mathrm{n} / \mathrm{s}$ ). The ${ }^{235} \mathrm{U}$ amount in two most sensitive fission chambers was $1.3 \mathrm{~g}$. The other two fission chambers with ${ }^{235} \mathrm{U}$ contained $0.01 \mathrm{~g}$ of fissile material with $\sim 130$ times lesser sensitivity than most sensitive detectors. Two thresholds fission chambers contained 1.3 and $0.3 \mathrm{~g}{ }^{238} \mathrm{U}$ and their sensitivities were differing in 4 times than each other. With these detectors the measurements of neutron strength uncertainty have been obtained on the level of $\pm 13 \%$.

Also on JT-60U [10] and JET [11] tokamaks the timeresolved neutron yield has been measured with fission neutron chambers. A wide range of the neutron flux, typically 9 or 10 decades, has been measured. ${ }^{235} \mathrm{U}$ detector is used in the low and medium neutron yield discharges and the ${ }^{238} \mathrm{U}$ detector is used in the high neutron yield discharges such as high-power NB heating experiments.

\section{Delayed Neutrons of Nuclear Fission}

The fission products are usually unstable due to being relatively neutron-rich nuclei and many of them are subject to beta decay. This releases additional energy in the form of beta particles, antineutrinos and gamma rays. In some cases, available energy in a highly excited state is enough to emit neutrons (delayed neutrons) instead of gamma emissions.

For example, ${ }^{235} \mathrm{U}$ absorbs thermal neutron and forms ${ }^{236} \mathrm{U}$ nucleus which fissions into two large fission fragments (Eq. 1). There are several hundreds of ways for the ${ }^{236} \mathrm{U}$ compound nucleus to fission. These fragments emit, on average, two or three free neutrons (in average 2.47), called prompt neutrons, for example:

${ }_{92}^{235} \mathrm{U}+\mathrm{n} \rightarrow{ }_{92}^{236} \mathrm{U}^{*} \rightarrow{ }_{36}^{90} \mathrm{Kr} \rightarrow{ }_{56}^{143} \mathrm{Ba}+3 \mathrm{n}$

The primary fission fragments $\left({ }^{90} \mathrm{Kr}\right.$ and $\left.{ }^{143} \mathrm{Ba}\right)$ have excess of neutrons as compared to the stable nuclei with the same atomic number. In most cases they undergo several successive $\beta^{-}$transitions to adjust their neutron/proton ratio. The fragment-pair above decays as follows:

$$
\begin{aligned}
& { }_{36}^{90} \mathrm{Kr} \underset{37}{\beta^{-}}{ }_{30}^{90} \mathrm{Rb} \stackrel{\beta^{-}}{\rightarrow}{ }_{38}^{90} \mathrm{Sr} \stackrel{\beta^{-}}{\rightarrow}{ }_{39}^{90} \mathrm{Y} \stackrel{\beta^{-}}{\rightarrow}{ }_{40}^{90} \mathrm{Zr} \text { (stable) } \\
& 33 \mathrm{~s} \quad 2.7 \mathrm{~min} \quad 28 \mathrm{y} \quad 64 \mathrm{~h}
\end{aligned}
$$

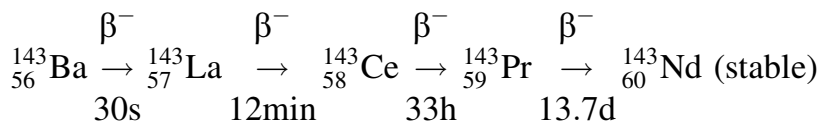

Some fission fragments occasionally undergo a stage of radioactive decay that yields a new nucleus in an excited state that emits an additional neutron, called a delayed neutron, to get to ground state, e.g.:

${ }_{35}^{87} \mathrm{Br}_{52} \stackrel{\beta^{-}}{\rightarrow}{ }_{36}^{87} \mathrm{Kr}_{51}^{*} \rightarrow{ }_{36}^{86} \mathrm{Kr}_{50}+\mathrm{n}$

These fission fragments which emit the neutron are called delayed-neutron precursor atoms. More than $99 \%$ of prompt neutrons are emitted very shortly (within $10^{-12}$ s) after the fission. Delayed neutrons are emitted long after the fission (sometimes several minutes later) with a yield of about $1 \%$. The decay chain is summarized as follows:

${ }_{\mathrm{Z}}^{\mathrm{A}} \mathrm{X}_{\mathrm{N}} \stackrel{\beta^{-}}{\rightarrow}{ }_{\mathrm{Z}+1}^{\mathrm{A}} \mathrm{Y}_{\mathrm{N}-1}^{*} \rightarrow \underset{\mathrm{Z}+1}{\mathrm{~A}} \mathrm{Y}_{\mathrm{N}-2}+\mathrm{n}($ delayed neutron $)$

The $\mathrm{X}$ nucleus is a delayed-neutron precursor, and the $\mathrm{Y}$ nucleus is a delayed-neutron emitter. Obviously, for these neutrons the 'delay time' is determined by the half-life of the precursor nucleus $\mathrm{X}$, which can be quite large, since the $\beta^{-}$decay is governed by the weak interaction. The main precursors of delayed neutrons during fission reaction are collected in Table 2 with the corresponding half-lives and abundances [12].

\section{Properties and Parameters of Delayed Neutrons}

An interesting consequence of the decay chain is that the excitation energy of the delayed-neutron emitter nucleus is usually much lower than the excitation energies of the direct fission fragments. Therefore, the average energy of the delayed neutrons is also smaller $(300 \div 600 \mathrm{keV})$ than that of the prompt neutrons ( $2 \mathrm{MeV}$ in average).

Examples of energy distributions of the delayed neutrons emitted after fission in ${ }^{232} \mathrm{Th},{ }^{238} \mathrm{U}$ and ${ }^{235} \mathrm{U}$ calculated using MCNP [13, 14] code are presented in Fig. 3. The simulations have been performed for flat cylinders $(2 \mathrm{R}=1.8 \mathrm{~cm}, \mathrm{H}=0.25 \mathrm{~cm})$ of the above pure isotopes, irradiated by the $2.45 \mathrm{MeV}$ neutrons. Delayed neutrons emitted after $10 \mathrm{~s}$ of the irradiation have been counted. The current of neutrons through the target surface has been obtained, containing neutrons of energy from $10^{-10} \mathrm{MeV}$ to $2.5 \mathrm{MeV}$. The "Normalized emission" label in the plots means the neutron current through the target surface in a given neutron energy interval normalized to the total 
Table 2 The main fission fragments (precursors) of delayed neutrons

\begin{tabular}{lcl}
\hline Precursor & Half-life of the precursor nucleus $(\mathrm{s})$ & Precursor abundance \\
\hline Br-87 & 55.6 & 0.033 \\
$\mathrm{I}-137$ & 24.5 & 0.178 \\
$\mathrm{Br}-88$ & 16.3 & 0.111 \\
$\mathrm{I}-138$ & 6.46 & 0.046 \\
$\mathrm{Rb}-93$ & 5.93 & 0.024 \\
$\mathrm{Br}-89$ & 4.38 & 0.101 \\
$\mathrm{Rb}-94$ & 2.76 & 0.162 \\
$\mathrm{I}-139$ & 2.30 & 0.046 \\
$\mathrm{As}-85$ & 2.08 & 0.107 \\
$\mathrm{Y}-98 \mathrm{~m}$ & 2.00 & 0.088 \\
$\mathrm{Kr}-93$ & 1.29 & 0.0048 \\
$\mathrm{Cs}-144$ & 1.00 & 0.0070 \\
$\mathrm{I}-140$ & 0.86 & 0.0052 \\
Br-91 & 0.542 & 0.017 \\
$\mathrm{Rb}-95$ & 0.384 & 0.049 \\
Rb-96 & 0.203 & 0.017 \\
Rb-97 & 0.170 & 0.0052 \\
\hline
\end{tabular}

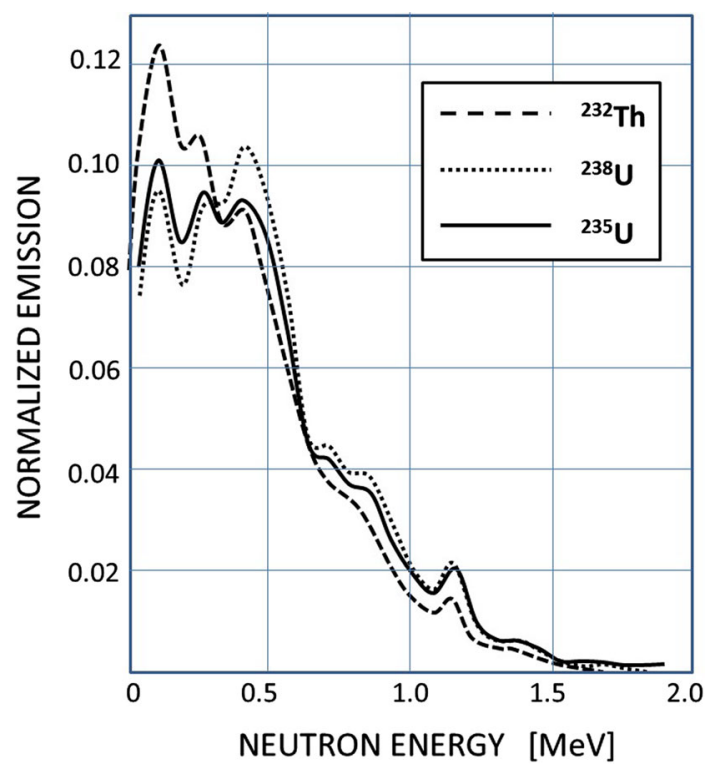

Fig. 3 Energy distributions of the delayed neutrons emitted after fissions in selected isotopes

current (in the whole energy range) per one starting source neutron.

More than 66 delayed neutron precursor nuclei are identified so far. Their half-lives range between 0.12 and $78 \mathrm{~s}$, therefore their delayed neutrons appear with considerably differing delay times. The time after which the delayed neutron appears is determined by the life of the precursor. Observation of delayed neutrons as a function of time shows their multi-exponential nature.

Among variety of possible mathematical representations of the delayed neutrons decay, a six-group model is commonly used $[15,16]$. Tables 3 and 4 present parameters of 6 group model of delayed neutrons after irradiation of ${ }^{238} \mathrm{U}$ and ${ }^{232} \mathrm{Th}$ by neutrons with energy of about $2 \mathrm{MeV}$ [12]. The corresponding decay curves and its distribution into 6 exponents with different decay constants are shown in Fig. $4 a$, b. It is evident that the curves for different isotopes slightly differ from each other (see the summary shown in the Fig. $4 c$ for ${ }^{238} \mathrm{U}$ and ${ }^{232} \mathrm{Th}$ ).

A general decay curve describing the number of particles (neutrons) after time $t$ is an exponential function:

$N(t)=N(0) e^{-\lambda t}$

where $N(0)$ is the number of particles in $t=0$ and $\lambda$ is the decay constant.

In order to obtain the decay rate of particles, one should differentiate Eq. (5) over time:

$\frac{d N(t)}{d t}=-\lambda N(0) e^{-\lambda t}$

Based on the above relationship the decay rate $R(t)$ of delayed neutrons can be written as:

$R(t)=\frac{d N}{d t}=C \sum_{i=1}^{n} \beta_{i} \lambda_{i} e^{-\lambda_{i} t}$

where $\beta_{\mathrm{i}}$-contribution of delayed neutrons for the $i$ th group of precursors, $\lambda_{\mathrm{i}}$ - decay constant of the $i$ th group of delayed neutrons, $n$-number of groups, $C$ - constant. 
Table 3 Parameters of 6 group model of delayed neutrons after irradiation of ${ }^{238} \mathrm{U}$ by neutrons with energy of about $2 \mathrm{MeV}$. Number of neutrons per one fission event, $v=2.805$, number of delayed neutrons per one fission event, $v_{d}=0.0460$

\begin{tabular}{lccc}
\hline Number of a group $i$ & Time of a half-life $\left(T_{1 / 2}\right)_{i}(\mathrm{~s})$ & Decay constant $\lambda_{i}(1 / \mathrm{s}) \lambda_{i}=\ln (2) /\left(\mathrm{T}_{1 / 2}\right)_{i}$ & Relative abundance $\beta_{i}$ \\
\hline 1 & $52.38 \pm 1.29$ & $0.01323 \pm 0.00033$ & $0.013 \pm 0.003$ \\
2 & $21.58 \pm 0.39$ & $0.03212 \pm 0.00043$ & $0.137 \pm 0.005$ \\
3 & $5.00 \pm 0.19$ & $0.1386 \pm 0.00537$ & $0.162 \pm 0.016$ \\
4 & $1.93 \pm 0.07$ & $0.359 \pm 0.013$ & $0.388 \pm 0.007$ \\
5 & $0.49 \pm 0.23$ & $1.415 \pm 0.066$ & $0.225 \pm 0.008$ \\
6 & $0.172 \pm 0.009$ & $4.03 \pm 0.21$ & $0.075 \pm 0.003$ \\
\hline
\end{tabular}

Table 4 Parameters of 6 group model of delayed neutrons after irradiation of ${ }^{232} \mathrm{Th}$ by neutrons with energy of about $2 \mathrm{MeV}$. Number of neutrons per one fission event, $v=2.616$, number of delayed neutrons per one fission event, $v_{\mathrm{d}}=0.0531$

\begin{tabular}{lccc}
\hline Number of a group $i$ & Time of a half-life $\left(T_{1 / 2}\right)_{i}(\mathrm{~s})$ & Decay constant $\lambda_{i}(1 / \mathrm{s}) \lambda_{i}=\ln (2) /\left(\mathrm{T}_{1 / 2}\right)_{i}$ & Relative abundance $\beta_{i}$ \\
\hline 1 & $56.03 \pm 0.95$ & $0.01237 \pm 0.00021$ & $0.034 \pm 0.002$ \\
2 & $20.75 \pm 0.66$ & $0.0334 \pm 0.0011$ & $0.150 \pm 0.005$ \\
3 & $5.74 \pm 0.24$ & $0.1208 \pm 0.0050$ & $0.155 \pm 0.021$ \\
4 & $2.16 \pm 0.08$ & $0.3209 \pm 0.012$ & $0.446 \pm 0.015$ \\
5 & $0.571 \pm 0.042$ & $1.2139 \pm 0.089$ & $0.172 \pm 0.013$ \\
6 & $0.211 \pm 0.019$ & $3.285 \pm 0.30$ & $0.043 \pm 0.006$ \\
\hline
\end{tabular}

\section{Activation Method of Neutron Measurements Based on Delayed Neutrons}

The activation method using delayed neutrons has been successfully applied in large fusion devices [11, 13, 17-19]. This variant of the activation method is implemented on JET together with the standard activation method based on gamma-spectrometry. The measurement set-up consists of a steal vessel with cadmium shield filled with polythene moderator ( $40 \mathrm{~cm}$ in diameter). The central hole is used to enter the activated sample through the pneumatic rabbit system end. The six ${ }^{3} \mathrm{He}$ proportional counters (one-inch diameter, $15 \mathrm{~cm}$ sensitive length) surround the hole (at a radial distance of $8 \mathrm{~cm}$ ), forming a hexagonal array. [19].

Unlike the indium used in gamma-spectrometry based activation method, the fissionable samples can be irradiated in each plasma discharge, one after another, due to short half-lives of delayed neutron precursors. In the system at JET [19], the sample of fission material is positioned in the irradiation end before the discharge and removed few seconds after the discharge is terminated. The rabbit system transfers the activates sample to the measurement set-up in $15 \mathrm{~s}$. The counting of the detectors starts together with discharge start and sampled at $500 \mathrm{~ms}$ intervals, after sample arrival the neutron signal is registered. Such a system gives information about the integral number of delayed neutrons, and the time decay curve was not analyzed. In the treatment of the ${ }^{238} \mathrm{U}$ measurements, the $18 \%$ contribution of the small ${ }^{235} \mathrm{U}$ contamination $(0.4 \%)$ has been taken into account. The detection efficiency has been established to be $(14.6 \pm 0.28) \%$ for ${ }^{238} \mathrm{U}$.

In the Institute of Nuclear Physics PAN, an experimental set-up for delayed neutron measurements was built and dedicated to activation methods applicable for fusion devices. A measuring chamber was designed, optimized with use of Monte Carlo simulations, and constructed [13, 20-22]. The device (called DET-12) is equipped with the set of twelve neutron detectors and the relevant system of data acquisition and digitizer allowing to determine the time decay curve of delayed neutrons. Figure 5 shows a general view of the DET-12 device and its components are specified in Fig. 6. The construction and dimensions of the individual components have been optimized in order to obtain the best performance of the device and the best possible signal-to-noise ratio. The background has been eliminated both from the scattered neutrons inside the device and from neutrons coming from the outside.

In the measuring system of the DET-12 device, the GaGe Octopus 8240CompuScope digitizer card was used. (http://www.gage-applied.com/digitizers/12-bit/pci/compu scope-octopus-82xx.htm, 22.05.2018). The card acts as a 
Fig. 4 Time decay curves of delayed neutrons from ${ }^{238} \mathrm{U}$ (a) and ${ }^{232} \mathrm{Th}$ (b) fission by $2 \mathrm{MeV}$ neutrons. Decay curves from each group (1-6) of delayed neutrons are marked. c Total decay curves of delayed neutrons for ${ }^{238} \mathrm{U}$ (a) and ${ }^{232} \mathrm{Th}$ are presented together for the visualization of differences in decay rates
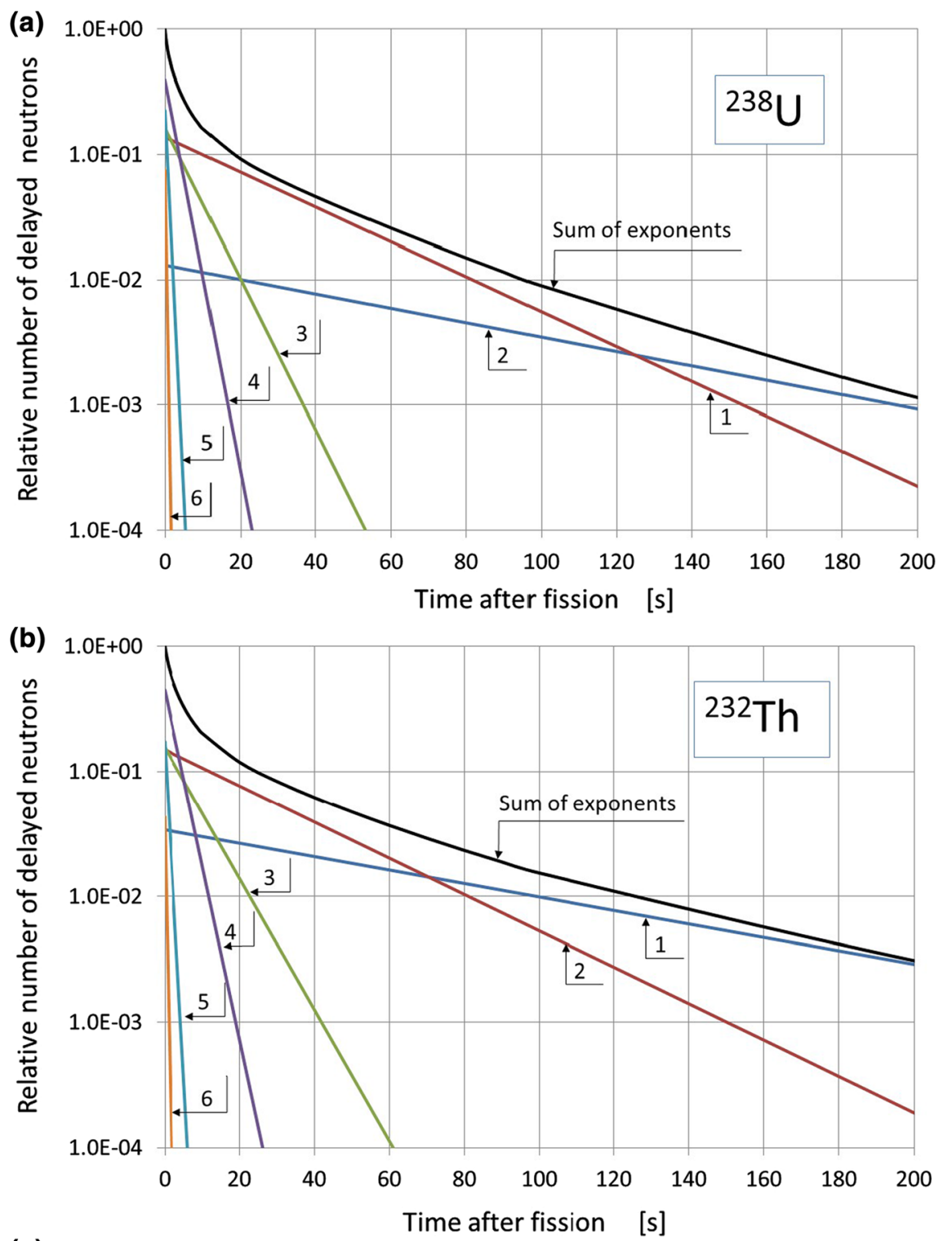

(c)

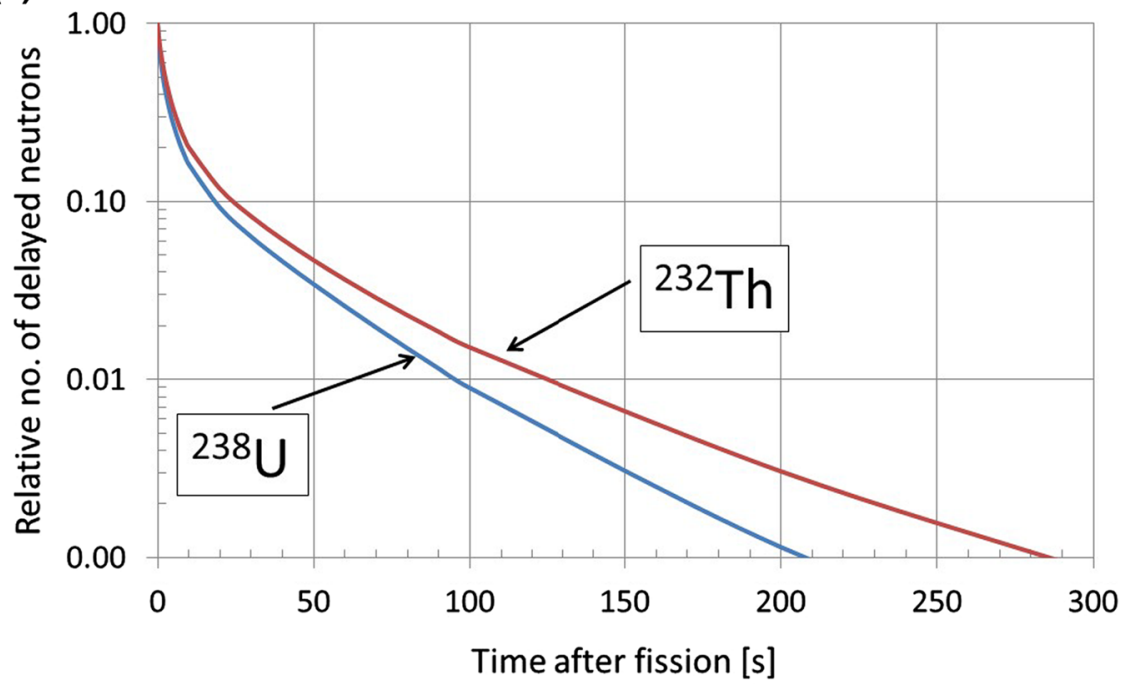


Fig. 5 General view of the DET-12 device for delayed neutrons measurements from fissionable samples. a Sample and detectors container (white), external electronics (on the right), b Container without the top cover
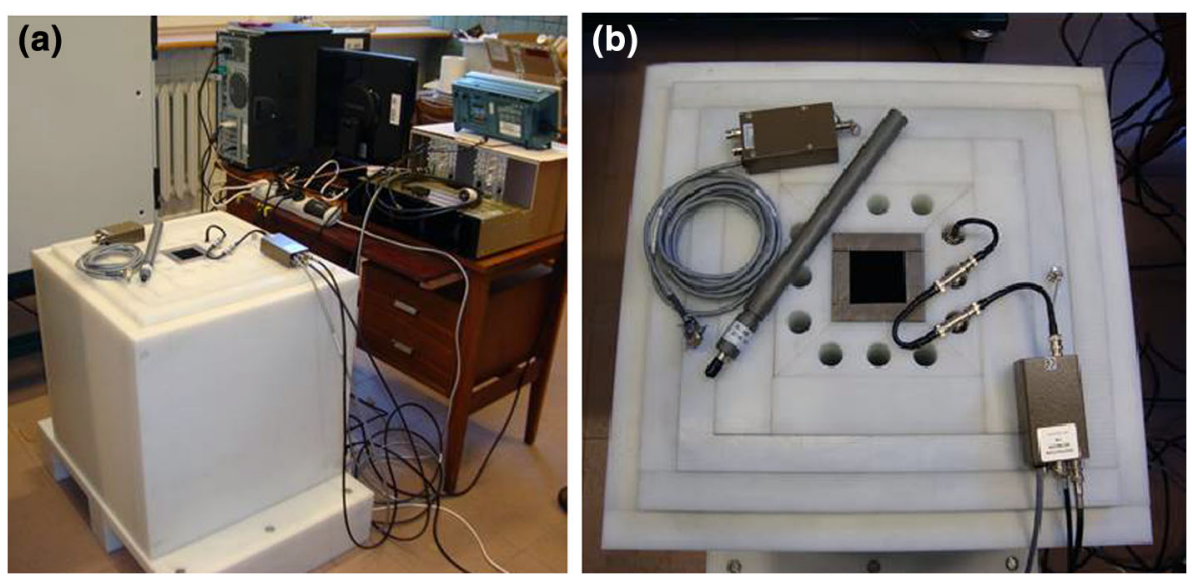

Fig. 6 Schematic cross section of the DET-12 setup for the delayed neutron detection (layers not in scale). 1 Borated polyethylene, 2 Cadmium layer, 3 Boron Carbide, 4

Polyethylene, 5 Detector holder, 6 Bismuth, 7 empty space (air), 8 Irradiated sample position

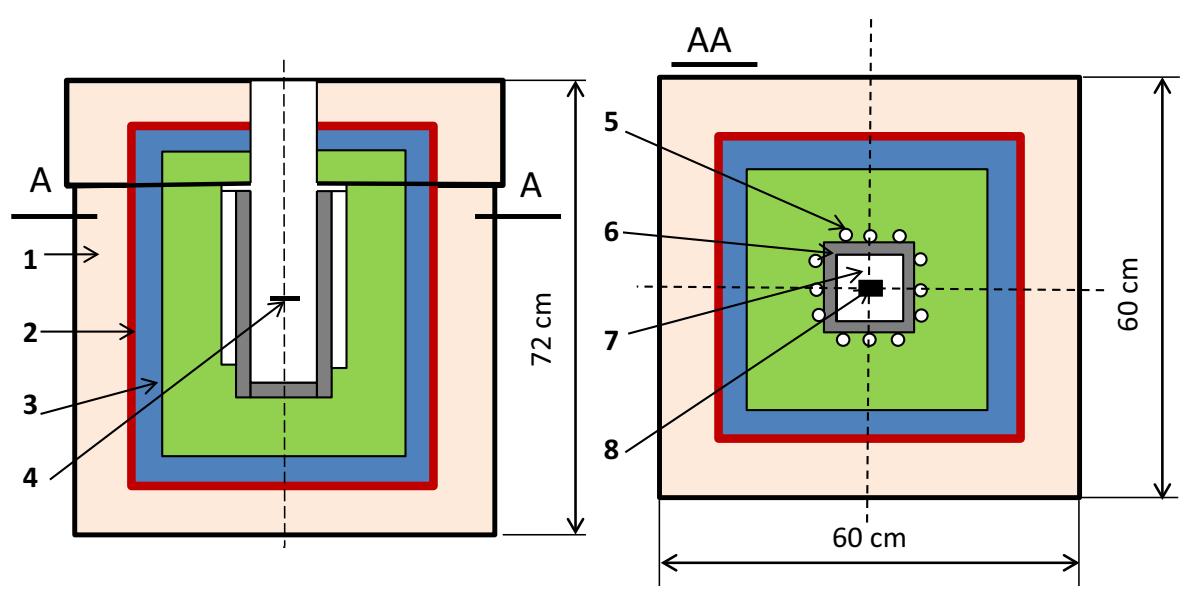

recorder of all impulses from helium detectors. It allows to record the shape of pulses, register the amplitude and the time in which they were recorded (the so-called "time stamp", allowing to arrange the pulse according to its time of appearance from the moment of registration). Built-in extended memory allows to record all pulses with maximum sampling up to several hundred seconds from the start of the digitizer. This allows later to accurately analyze the pulses in terms of their appearance time and the ability to assign a given pulse to the time channel [23].

Time decay curve of delayed neutrons generated in the target-sample one can measure in time analyzer with $\Delta \mathrm{t}$ channel width. Number of counts in channel $k$ one calculate from Eq. (7):

$N\left(t_{k}\right)=C \sum_{i=1}^{n} \beta_{i} \lambda_{i} \int_{t_{k}}^{t_{k}+\Delta t} e^{-\lambda_{i} t} d t$

this gives

$N\left(t_{k}\right)=C \sum_{i=1}^{n} \beta_{i}\left(1-e^{-\lambda_{i} \Delta t}\right) e^{-\lambda_{i} t_{k}}$ where $C=\varepsilon N_{0} P, \varepsilon$-detector efficiency, $N_{0}$-number of primary neutrons reaching the sample-target, $\left[\mathrm{n} / \mathrm{cm}^{2}\right], P$ material parameter: product of macroscopic fission cross section, $\Sigma_{\mathrm{f}}$, and number of delayed neutrons per one fission event, $v_{\mathrm{d}}$.

For the target-sample which is a single isotope (e.g. ${ }^{238} \mathrm{U}$ or $\left.{ }^{232} \mathrm{Th}\right)$ material parameter is:

$P=v_{d} \sigma_{f} \frac{N_{A}}{A} m$

where $\sigma_{\mathrm{f}}$-microscopic fission cross section $\left(\mathrm{cm}^{2}\right), \mathrm{N}_{\mathrm{A}}-$ Avogadro number, A-atomic number, $m$-mass of the sample-target $(\mathrm{g})$

The purpose of the measurement is to determine the number of neutrons falling on a sample. This number can be estimated from the number of counts in a single channel of the formula (9):

$N_{0}=\frac{N\left(t_{k}\right)}{\varepsilon P \sum_{i=1}^{n} \beta_{i}\left(1-e^{-\lambda_{i} \Delta t}\right) e^{-\lambda_{i} t_{k}}}$

The above-described constant $C$ corresponds to the ideal analytical solution describing the decay curve of 
Fig. 7 An example of the calculated delayed neutrons decay curve (Eq. 9) measured in time intervals $\Delta \mathrm{t}=1 \mathrm{~s}$ for ${ }^{238} \mathrm{U}$ sample

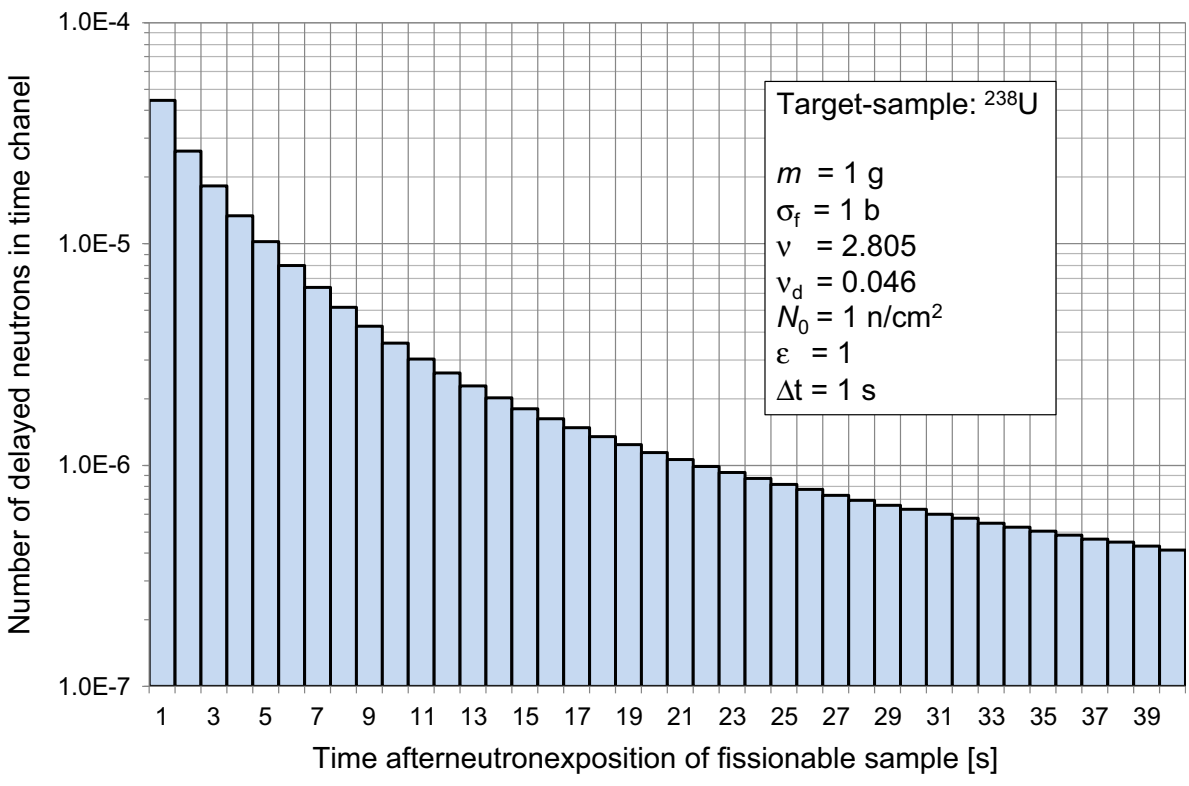

the delayed neutron flux. In fact, the calibration constant $C$ depends not only on the efficiency of detection, the macroscopic cross-section on the split, the size and detailed elemental composition of the target, but also on the entire experimental environment. For a specific application of the DET-12 system, it will be necessary to carry out detailed Monte Carlo numerical modeling that reproduces the measurement conditions at the fusion device, supplemented by calibration measurements. The latter can be carried out in laboratory conditions using an isotopic source. Detailed procedures related to the adaptation of DET-12 to the actual measurement conditions at the fusion device are presented in paper [23].

An example of the calculated decay curve registered in $\Delta \mathrm{t}=1 \mathrm{~s}$ channels obtained using the above formulae is presented in Fig. 7. A rapid drop in counts over time means that the measurement of delayed neutrons should be performed as soon as possible after exposure. This effect is a disadvantage (very fast drop in count statistics) and advantage (immediate experimental result, the registration of the decay curve takes few minutes at most) of the activation method using delayed neutrons. Target, which is irradiated with a primary neutron flux, is placed as close as possible to the tokamak's chamber and after the exposure has to be transported by pneumatic mail to the place of measurement. From this it follows that a certain delay between the measurement time and the exposure time is inevitable. On the other hand, the final result of the measurement is obtained very quickly after irradiation. This, compared to the classical activation method, is an advantage, as often isotopes used in classical activation method require a long cooling time before the final spectrometer gamma measurement.

\section{Summary}

Fission chambers and the activation method which uses delayed neutrons as measurement methods for determination of the yield of a fusion neutron source are successfully applied (and will be applied) in large fusion devices. The main feature of fission chambers are very small dimensions, a wide range of neutron yield which can be measured and extremely low backgrounds from gamma rays and from other competing reactions. The neutron delayed activation method can be a good method of detecting very high neutron fusion fluxes due to the relatively small number of delayed neutrons which are registered. Compared to the classical activation method, the final measurement result (fusion neutron fluence) can be obtained in a shorter time after the target has been activated. Targets for the delayed neutron method must be measured as soon as possible after irradiation. In the case of classical activation, it is often necessary to cool the target in order to extinguish the high activity of short-lived isotopes, which extends the total measurement time. Knowledge of the physics of the heavy elements fission phenomenon is undoubtedly very helpful in the selection of the right measurement way both in the case of fission chambers and of the activation method that use the presence of delayed neutrons.

Acknowledgements Lecture on Summer School of Plasma Diagnostics: PhDiaFusion 2017. 'Neutron and gamma diagnostics for fusion plasma'. 11-15 September 2017, Podlesice, Poland. https://phdia2017. ifj.edu.pl/. The work was performed as part of the Institute of Nuclear Physics, Polish Academy of Sciences Task Number S61414: 'Hightemperature plasma diagnostics'. Thank you very much to my colleague Eng. Andrzej Igielski for helpful discussions and comments during the preparation of the article. 
Open Access This article is distributed under the terms of the Creative Commons Attribution 4.0 International License (http://creative commons.org/licenses/by/4.0/), which permits unrestricted use, distribution, and reproduction in any medium, provided you give appropriate credit to the original author(s) and the source, provide a link to the Creative Commons license, and indicate if changes were made.

\section{References}

1. A.V. Krasilnikov, M. Sasao, YuA Kaschuck, T. Nishitani, P. Batistoni, V.S. Zaveryaev, S. Popovichev, T. Iguchi, O.N. Jarvis, J. Källne, C.L. Fiore, A.L. Roquemore, W.W. Heidbrink, R. Fisher, G. Gorini, D.V. Prosvirin, AYu. Tsutskikh, A.J.H. Donné, A.E. Costley, C.I. Walker, Nucl. Fusion 45, 1503 (2005). https:// doi.org/10.1088/0029-5515/45/12/005

2. S.F. Mughabghab, Atlas of Neutron Resonances, 5 th edn. (Elsevier, Amsterdam, 2006)

3. K.H. Beckurts, K. Wirtz, Neutron Physics (Springer, Berlin, 1964). ISBN 978-3-642-87614-1

4. A.M. Weinberg, E.P. Wigner, The Physical Theory of Neutron Chain Reactors (The University of Chicago Press, Chicago, 1958)

5. G.D. James, J.E. Lynn, A. Michaudon, Nuclear Fission and Neutron-induced Fission Cross-sections (Pergamon Press, Oxford, 1981). ISBN 9781483189765

6. ENDF-6 Formats Manual Data Formats and Procedures for the Evaluated Nuclear Data Files ENDF/B-VI and ENDF/B-VII and https://www-nds.iaea.org/exfor/endf.htm.

7. G.F. Knoll, Radiation Detection and Measurement, 3rd edn. (Wiley, Hoboken, 2000)

8. M. Ishikawa, T. Kondoh, T. Nishitani, Y. Kusama, J. Plasma Fusion Res. Ser. 8, 334-337 (2009)

9. H.W. Hendel, R.W. Palladino, Cris W. Barnes, M. Diesso, J.S. Felt, D.L. Jassby, L.C. Johnson, L.P. Ku, Q.P. Liu, R.W. Motley, H.B. Murphy, J. Murphy, E.B. Nieschmidt, J.A. Roberts, T. Saito, J.D. Strachan, R.J. Waszazak, K.M. Young, Rev. Sci. Instrum. 61, 1900 (1990). https://doi.org/10.1063/1.1141115

10. T. Hayashi, T. Nishitani, A.M. Sukegawa, M. Ishikawa, K. Shinohara, Rev. Sci. Instrum. 79, 10E506 (2008)

11. O.N. Jarvis, Plasma Phys. Control. Fusion 36, 209 (1994). https:// doi.org/10.1088/0741-3335/36/2/002

12. Delayed Neutron Data for the Major Actinides. A report by the Working Party on International Evaluation Co-operation of the NEA Nuclear Science Committee, NEA/WPEC-6, OECD PUBLICATIONS. https://www.oecd-nea.org/science/wpec/volume6/ volume6.pdf. Accessed 2002
13. B. Bieńkowska, K. Drozdowicz, B. Gabańska, A. Igielski, R. Prokopowicz, U. Wiącek, U. Woźnicka, Physical assumptions for a design of the DET-12 chamber for detection of delayed neutrons. Rept. IFJ No. 2074/AP, Kraków, 2014. https://www.ifj.edu. pl/publ/reports/2014/2074.pdf?lang=pl. Accessed 2002

14. X-5 Monte Carlo Team, MCNP: a general Monte Carlo N-particle transport code. Version 5, Rept. LA-UR-03-1987, Los Alamos National Laboratory, 2003

15. G.R. Keepin, T.F. Wiemett, R.K. Zeigler, Phys. Rev. 107(4), 1044 (1957)

16. G.R. Keepin, Physics of Nuclear Reactors (Addison-Wesley Publ. Co., Massachusetts, 1965)

17. M.T. Swinhoe, O.N. Jarvis, Nucl. Instrum. Meth. Phys. Res. 221(2), 460 (1984). https://doi.org/10.1016/0167-5087(84)900206

18. H. Tourwe, A. Fabry, P. D'hondt, Delayed neutron counting system for JET plasma neutron yields diagnostics, in Reactor Dosimetry, ed. by J.P. Genthon, H. Röttger (Springer, Dordrecht, 1985), pp. 309-315. https://doi.org/10.1007/978-94-009-5378-9_ 30

19. P. van Belle, O.N. Jarvis, G. Sadler, S. de Leeuw, P. D'Hondt, M. Pillon, Rev. Sci. Instr. 61, 3178 (1990). https://doi.org/10.1063/1. 1141679

20. G. Tracz, B. Bieńkowska, K. Drozdowicz: Detection of delayed neutrons from neutron activation of fissionable substance samples. Monte Carlo modelling of response of the DET-12 device. Rept. IFJ No. 2061/PN, Kraków, 2013. https://www.ifj.edu.pl/ publ/reports/2014/2061.pdf?lang=pl. Accessed 2002

21. B. Bieńkowska, K. Drozdowicz, B. Gabańska, A. Igielski, W. Janik, A. Kurowski, G. Tracz, U. Wiącek, J. Dankowski, Technical design and operation tests of the DET-12 device for detection of delayed neutrons. Rept. IFJ No. 2075/AP, Kraków, 2014. https://www.ifj.edu.pl/publ/reports/2014/2075.pdf?lang=pl. Accessed 2002

22. A. Kurowski, B. Bieńkowska, K. Drozdowicz, B. Gabańska, A. Igielski, R. Prokopowicz, G. Tracz, U. Wiącek, U. Woźnicka, J. Fusion Energ. 37, 120-123 (2018). https://doi.org/10.1007/ s10894-018-0156-3

23. U. Wiącek, U. Woźnicka, J. Fusion Energ. 37, 120-123 (2018). https://doi.org/10.1007/s10894-018-0156-3

24. A. Kurowski, The method of measuring neutron fluxes from the synthesis reaction in thermonuclear reactors by detecting delayed neutrons from the activation of fissile materials. Design and construction of the measurement system. Ph.D. thesis (in Polish), Institute of Nuclear Physics, Polish Academy of Sciences, Kraków, 2018 